\title{
Effects of different intraabdominal pressure levels on oxidative stress markers in laparoscopic cholecystectomy
}

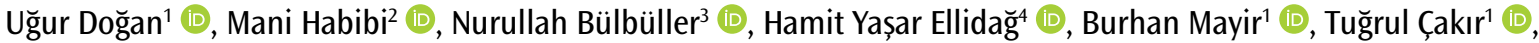

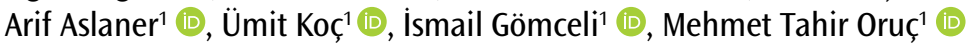

ABSTRACT Objective: To evaluate the effect of laparoscopic cholecystectomy performed under different intraabdominal pressure on oxidative stress markers.

Material and Methods: This prospective, randomized, controlled study examined 90 consecutive healthy patients who underwent elective laparoscopic cholecystectomy with the diagnosis of symptomatic cholelithiasis. The patients were divided into three groups, 30 patients in each. Group 1 included patients who underwent laparoscopic cholecystectomy at a $\mathrm{CO}_{2}$ pneumoperitoneum pressure of $7 \mathrm{mmHg}$, Group 2 patients who underwent laparoscopic cholecystectomy at a $\mathrm{CO}_{2}$ pneumoperitoneum pressure of $10 \mathrm{mmHg}$, and Group 3 patients who underwent laparoscopic cholecystectomy at a $\mathrm{CO}_{2}$ pneumoperitoneum pressure of $13 \mathrm{mmHg}$. Blood samples were collected preoperatively, perioperatively, and postoperatively for measurement of the serum levels of ischemia modified albumin and an analysis of total antioxidant status and total oxidant status. Intra-group comparisons were made.

ORCID IDs of the authors: U.D. 0000-0001-8097-3168; M.H. 0000-0003-3420-2762; N.B. 0000-0001-6126-0808; H.Y.E. 0000-0002-7511-2547; B.M. 0000-0001-8428-6461; T.C. 0000-0001-7952-8462; A.A. 0000-0001-7346-6977; U.K. 0000-0001-9055-6675; I.G. 0000-0001-6734-1254; M.T.0.0000-0002-6051-691X.

Cite this paper as: Doğan U, Habibi M, Bulbuller N, Ellidağ HY, Mayir B, Çakır $T$, et al. Effects of different intraabdominal pressure levels on oxidative stress markers in laparoscopic cholecystectomy. Turk J Surg 201834 (3): 212-216.

'Department of General Surgery, Antalya Training and Research Hospital, Antalya, Turkey 2Department of General Surgery Alanya Training and Research Hospital, Antalya, Turkey

${ }^{3}$ Department of General Surgery, Akdeniz University School of Medicine, Antalya, Turkey

${ }^{4}$ Central Laboratories, Antalya Training and Research Hospital, Antalya, Turkey

This study was presented at the $23^{\text {rd }}$ International Congress of the European Association for Endoscopic Surgery (EAES), 3-6 June 2015, Bucharest, Romania.

\section{Corresponding Author} Uğur Doğan

e-mail:ugurdoga1@hotmail.com

Received: 22.05.2017

Accepted: 24.10.2017

CCopyright 2018

by Turkish Surgical Association

Available online at

www.turkjsurg.com
Results: Group 1 experienced a significant increase in the postoperative ischemia modified albumin values compared to preoperative ischemia modified albumin values $(p=0.013)$. Group 2 experienced a significant decrease in the perioperative total antioxidant status values compared to preoperative and postoperative total antioxidant status values $(p=0.009)$. Group 3 experienced a significant increase in the perioperative total oxidant status and oxidative stress index values compared to preoperative values $(p<0.001)$. Group 3 experienced a significant increase in the perioperative and postoperative ischemia modified albumin values compared to preoperative values $(p<0.001)$.

Conclusion: Increased levels of oxidative stress markers were detected in patients who underwent laparoscopic cholecystectomy at a high intraabdominal pressure level.

Keywords: Antioxidant, intraabdominal pressure, laparoscopic cholecystectomy, oxidative stress, pneumoperitoneum

\section{INTRODUCTION}

Laparoscopic cholecystectomy (LC) is a minimally invasive surgical procedure considered the gold standard and first choice in the treatment of symptomatic gallstones. This procedure has several advantages compared to open surgery, such as less pain and scarring and early return to activity (1). Nevertheless, adverse hemodynamic changes may occur with LC, including the increase of intraabdominal pressure (IAP). Besides the many advantages of LC, there are also risks, such as bile duct injury, bile leakage, intraabdominal organ and vascular injuries, perfusion defects due to rising IAP and oxidative tissue damage. Inappropriate pneumoperitoneum (PP) pressures can create life-threatening risks that are often not recognized, especially in elderly patients with chronic diseases. An increase in IAP depends on the presence of $\mathrm{PP}$, which increases the systemic vascular resistance and decreases venous return, cardiac output, and stroke volume. Depending on the extent of PP, visceral perfusion, splanchnic perfusion, portal venous flow, and renal blood flow may also decrease (2-4). The reduction of abdominal visceral perfusion during laparoscopic surgical procedures increases oxidative stress $(5,6)$, which increases the production of lipid and protein oxidation products and decreases the production of antioxidant enzymes and circulation of vitamins.

There is a balance between oxidants and antioxidants that must be constantly controlled so that the biochemical functions in living beings may continue. Oxidative damage may occur when there is an imbalance in favor of oxidants. Some cellular and extracellular substances against the oxidative stress in the human body react with the free radicals, creating an imbalance between the oxidant and antioxidant levels, which is the main cause of tissue damage. There are many markers for assessing oxidative stress and antioxidant status. However, it is both time consuming and costly to measure these markers separately. To assess the risk of such a damage, the total antioxidant status (TAS) and total oxidant status (TOS) can be measured using a fully automated method $(7,8)$. Formation of the free radicals as a result of oxidative stress and hypoxia reduces the binding capacity for transition metals in the human $\mathrm{N}$-terminal region of albumin, leading to the production of ischemia modified albumin (IMA). The IMA level can help in assessing the blood flow changes that may occur in the splanchnic area. The oxidant 
and antioxidant levels can be demonstrated by the TAS, TOS, and IMA measurements appropriately and correctly $(7,8)$.

The LC procedure still shows diversity and differences due to the diversity of surgical instruments and techniques used, the number of trocars used, and the differences in the PP pressure created. During a LC procedure, keeping the PP pressure at the levels as ideal as possible without jeopardizing operational safety will ensure that the patient is protected from possible complications such as oxidative stress. Our aim in this study is to show the possible oxidative effects of the PP pressure and to draw attention to this issue. In our study, we formed groups of patients who underwent operation at low, medium, and high pressures, assuming that the $10-12 \mathrm{mmHg}$ PP pressure was created while performing LC in the general practice. We performed surgical operations under 7,10 , and $13 \mathrm{mmHg}$ PP pressures and investigated the effect of pressure differences on oxidative stress. Despite the importance of the IAP in LC, no study, to the best of our knowledge, has assessed the risk of performing LC under different pressures. To fill this research gap, our study examined the effect of LC performed under different IAP levels on oxidative stress markers.

\section{MATERIAL AND METHODS}

\section{Patients}

This prospective, randomized, and controlled study examined 90 consecutive healthy patients who underwent elective LC with the diagnosis of symptomatic cholelithiasis between June 2014 and April 2015. Patients with neoplastic diseases, diabetes mellitus, inflammatory disease, biliary pancreatitis, liver dysfunction, antihypertensive treatment, ischemic heart disease, chronic renal failure, or congestive heart failure were excluded. The approval for the study protocol was obtained from the local ethics committee of the Antalya Training and Research Hospital (2013-162). The study was conducted in accordance with the Declaration of Helsinki, and all study participants provided written informed consent before the study.

The 90 patients were divided into three groups, 30 patients in each. Group 1 included patients who underwent LC at a $\mathrm{CO}_{2}$ PP pressure of $7 \mathrm{mmHg}$, Group 2 patients who underwent LC at a $\mathrm{CO}_{2}$ PP pressure of $10 \mathrm{mmHg}$, and Group 3 patients who underwent $\mathrm{LC}$ at a $\mathrm{CO}_{2}$ PP pressure of $13 \mathrm{mmHg}$. Preoperative ( 1 hour before the surgery), perioperative (20 minutes after the pressure generated PP), and postoperative (postoperative 24th hours) blood samples were taken from the patient groups. The samples were centrifuged at $3000 \mathrm{rpm}$ for $15 \mathrm{~min}$ and stored in Eppendorf tubes at $-80^{\circ} \mathrm{C}$. The TAS, TOS, IMA, IMA-to-serum albumin ratio (IMAR), oxidative stress index (OSI), and albumin values were measured in serum. Intragroup comparisons were made following preoperative, perioperative, and postoperative measurements. A power analysis was performed. Preoperative and perioperative values were compared using the mean and standard deviation values. The power value of the analysis of the difference between the two values was found to be 0.999 according to the significance level of 0.05 and the effect size of 1.14 .

\section{Calculation of the TAS}

TAS was measured with a Beckman AU5800 autoanalyzer (Beckman Coulter Diagnostics, BreaCA, USA), using the Erel method. In this colorimetric method, the presence of antioxidants causes a loss of color in dark-blue-green-colored ABTS, with the color change recorded at $660 \mathrm{~nm}$ and the results given in micromolar trolox liters. The measurement is calibrated with trolox equivalent vitamin $\mathrm{E}$. The method's coefficient of variation (CV) has been reported to be $<5 \%$ (7).

\section{Calculation of the TOS}

Total oxidant status was measured with a Beckman AU5800 auto analyzer (Beckman Coulter Diagnostics, BreaCA, USA) using the Erel method. This colorimetric method is based on the oxidization of ferrous-ion iron complex to ferric ion, which creates a colored compound in an acidic medium. The measurement is calibrated with $\mathrm{H}_{2} \mathrm{O}_{2}$ and the results given in $\mu$ mol $\mathrm{H}_{2} \mathrm{O}_{2}$ Equiv./L. The method's CV has been reported to be $<5 \%$ (8).

\section{Calculation of the Oxidative Stress Index}

The percentage ratio of TOS to TAS was accepted as the OSI (9). To determine the ratio, the resulting micromolar unit of TAS was changed to $\mathrm{mmol} / \mathrm{L}$, and the OSI value was calculated according to the following equation: OSI (arbitrary unit)=TOS (micromolar hydrogen peroxide equivalent/L)/TAS (micromolar trolox equivalent/L).

\section{Measurement of the Ischemic Modified Albumin Level}

After adding $50 \mu \mathrm{l}$ of a $0.1 \%$ cobalt chloride solution to $200 \mu \mathrm{l}$ of serum, the mixture was vortexed and then incubated for 10 min to induce the albumin-cobalt binding. After incubation, $50 \mu \mathrm{l}$ of $1.5 \mathrm{mg} / \mathrm{mL}$ dithiothreitol (DTT) solution was added to form a color reaction with unbound cobalt and albumin, which typically occurs within $2 \mathrm{~min}$. After $2 \mathrm{~min}$, the reaction was completed by adding $1 \mathrm{~mL}$ of $0.9 \% \mathrm{NaCl}$ to the mixture. The same steps were performed simultaneously to prepare a blind sample by using distilled water instead of DTT. After the reactions had been completed, the absorbance values of the experimental and blind samples as read at $470 \mathrm{~nm}$ were recorded as the IMA values (10). The equation IMA value/individual serum albumin concentration was used to maintain the IMAR to avoid introducing the confounding factor of the impact of albumin concentration differences between groups.

\section{Statistical Analysis}

Descriptive statistics were presented in terms of the frequency, percentage, mean, and standard deviation (SD) of the variables. In the test of normality, the Shapiro-Wilks test was performed if the sample size was $<50$, and the KolmogorovSmirnov test if the sample size was $\geq 50$. The Kruskal-Wallis test was performed for non-parametric comparison of the three patient groups. In cases when the normal distribution was assumed, the analysis of variance was performed for comparison of the three groups, and the Turkey test for comparison of two groups. For the preoperative, perioperative, and postoperative values of the measurements for each group, the Friedman test was performed if the measurements were not in agreement with normal distribution, and normal distribution variance analysis for repeated measurements was performed if the measurements were in agreement with normal distribution. When the differences between the measurements were found to be significant in the binary comparisons, the Bonferroni-Dunn and Bonferroni tests were performed as post hoc tests. P-values less than 0.05 were considered statistically significant. All analyses were performed using the Statisti- 
cal Packages for the Social Sciences (SPSS) version 20.0 (IBM Corp.; Armonk, NY, USA).

\section{RESULTS}

The patients were divided into three groups. Group 1 (30 patients who underwent $\mathrm{LC}$ under a $\mathrm{CO}_{2} \mathrm{PP}$ pressure of $7 \mathrm{~mm} \mathrm{Hg}$ ) consisted of 11 males and 19 females of a mean age of 36.46 years (range, 26-52 years). Group 2 (30 patients who underwent LC under a $\mathrm{CO}_{2}$ PP pressure of $10 \mathrm{~mm} \mathrm{Hg}$ ) consisted of 13 males and 17 females of a mean age of 43.63 years (range, 28-50 years). Group 3 (30 patients who underwent $L C$ under a $\mathrm{CO}_{2} \mathrm{PP}$ pressure of $13 \mathrm{~mm} \mathrm{Hg}$ ) consisted of 12 males and 18 females of a mean age of 43.33 years (range, 33-52 years). The surgical duration for Groups 1, 2, and 3 was 38.67 \pm 34.11 , $34.24 \pm 11.23$, and $32.53 \pm 34.46 \mathrm{~min}$, respectively. The difference in surgical duration among the groups was not found to be statistically significant $(p=0.078)$.

The serum levels of IMA and analysis of TAS and TOS were done preoperatively, perioperatively, and postoperatively for measurement, and intra-group comparisons were performed.

Table 1 shows preoperative, perioperative, and postoperative TAS, TOS, IMA, IMAR, OSI, and albumin values of the groups. Group 1 experienced a significant decrease in the postoperative albumin level and a significant increase in the postoperative IMA level compared to the preoperative level of these variables ( $p=0.009, p=0.013$, respectively). A significant increase was found in both the perioperative IMAR and postoperative IMAR values compared to the preoperative values of these variables ( $p=0.012, p=0.026$, respectively).

Group 2 only experienced a significant decrease in the perioperative TAS value, a difference that was found to arise from an intraoperative reduction $(p=0.009)$.

Group 3 experienced a significant increase in the perioperative albumin and postoperative albumin levels compared to the preoperative level $(p<0.001, p=0.002$, respectively). Group 3 experienced a significant increase in the perioperative TOS values compared to preoperative values and a significant decrease in the postoperative TOS values compared to perioperative values $(p<0.001, p=0.001$, respectively). Group 3 experienced a significant increase in the perioperative and postoperative IMA values compared to preoperative values $(p<0.001)$, a significant increase in the perioperative OSI values compared to preoperative values $(p<0.001)$, and a signifcant decrease in the postoperative OSI values compared to perioperative values $(p<0.001)$.

Table 2 shows intra-group binary comparisons.

\section{DISCUSSION}

Cholelithiasis is one of the most frequent diseases of the gastrointestinal system, with a $15 \%-20 \%$ overall prevalence in the population aged between 15 and 50 years (11). The gold standard treatment for cholelithiasis is LC, an effective and minimally invasive surgical technique (12). However, the use of this method poses a risk of complications because of an increased IAP, thrombotic disease due to decreases in the perfusion of the abdominal organs, a decrease in the vena cava
Table 1.TAS, TOS, IMA, IMAR, OSI, and albumin values of Groups 1,2 , and 3

\begin{tabular}{|c|c|c|c|c|}
\hline & $\begin{array}{c}\text { Preoperative } \\
\text { Value } \\
\text { Mean } \pm \text { SD }\end{array}$ & $\begin{array}{c}\text { Perioperative } \\
\text { Value } \\
\text { Mean } \pm \text { SD }\end{array}$ & $\begin{array}{c}\text { Postoperative } \\
\text { Value } \\
\text { Mean } \pm \text { SD }\end{array}$ & $p$ \\
\hline \multicolumn{5}{|l|}{ Group 1} \\
\hline TAS & $2.497 \pm 0.55$ & $2.563 \pm 0.38$ & $2.497 \pm 0.38$ & 0.455 \\
\hline TOS & $4.183 \pm 3.52$ & $4.933 \pm 5.14$ & $2.652 \pm 1.19$ & 0.846 \\
\hline OSI & $1.758 \pm 1.27$ & $1.854 \pm 1.73$ & $1.090 \pm 0.53$ & 0.786 \\
\hline IMA & $0.380 \pm 0.03$ & $0.393 \pm 0.02$ & $0.394 \pm 0.02$ & $0.011 *$ \\
\hline IMAR & $0.114 \pm 0.03$ & $0.118 \pm 0.01$ & $0.119 \pm 0.01$ & $0.006^{*}$ \\
\hline Albumin & $3.490 \pm 0.64$ & $3.357 \pm 0.31$ & $3.337 \pm 0.35$ & $0.001^{*}$ \\
\hline \multicolumn{5}{|l|}{ Group 2} \\
\hline TAS & $3,160 \pm 0.64$ & $2.861 \pm 0.54$ & $2.964 \pm 0.63$ & $0.007^{*}$ \\
\hline TOS & $3.543 \pm 2.83$ & $3.988 \pm 5.19$ & $2.965 \pm 2.04$ & 0.381 \\
\hline OSI & $1.085 \pm 0.80$ & $1.329 \pm 1.41$ & $1.012 \pm 0.61$ & 0.786 \\
\hline IMA & $0.371 \pm 0.02$ & $0.380 \pm 0.02$ & $0.377 \pm 0.02$ & 0.172 \\
\hline IMAR & $0.114 \pm 0.02$ & $0.117 \pm 0.01$ & $0.114 \pm 0.01$ & 0.356 \\
\hline Albumin & $3.365 \pm 0.54$ & $3.274 \pm 0.40$ & $3.343 \pm 0.32$ & 0.764 \\
\hline \multicolumn{5}{|l|}{ Group 3} \\
\hline TAS & $2.757 \pm 0.49$ & $2.726 \pm 0.49$ & $2.771 \pm 0.40$ & 0.649 \\
\hline TOS & $3.777 \pm 2.98$ & $4.090 \pm 3.26$ & $2.702 \pm 0.85$ & $0.001^{*}$ \\
\hline OSI & $1.368 \pm 0.92$ & $1.496 \pm 1.01$ & $1.150 \pm 0.91$ & $0.001^{*}$ \\
\hline IMA & $0.352 \pm 0.02$ & $0.384 \pm 0.02$ & $0.396 \pm 0.02$ & $0.001 *$ \\
\hline IMAR & $0.114 \pm 0.02$ & $0.117 \pm 0.01$ & $0.114 \pm 0.01$ & 0.106 \\
\hline Albumin & $3.160 \pm 0.47$ & $3.307 \pm 0.40$ & $3.493 \pm 0.34$ & $0.002^{*}$ \\
\hline
\end{tabular}

*Significant difference, $\mathrm{p}<0.05$

TAS: Total antioxidant status (mmol Trolox Equiv./L); TOS: Total oxidant status ( $\mu$ molH2O2Equiv./L); IMA: Ischemia modified albumin (ABSU); Albumin (g/dL); OSI: Oxidative stress index; and IMAR: IMA-to-serum albumin ratio (arbitrary unit)

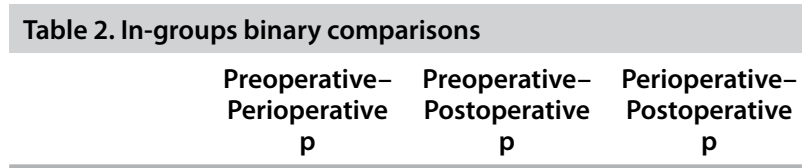

$\begin{array}{llll}\text { Group 1 } & & & \\ \text { IMA } & 0.078 & 0.013^{*} & 0.999 \\ \text { IMAR } & 0.012^{*} & 0.026^{*} & 0.999 \\ \text { Albumin } & 0.054 & 0.009^{*} & 0.999\end{array}$

Group 2

$\begin{array}{llll}\text { TAS } & 0.009^{*} & 0.054 & 0.999 \\ \text { Group 3 } & & & \\ \text { Albumin } & 0.001^{*} & 0.002^{*} & 0.072 \\ \text { TOS } & 0.001^{*} & 0.999 & 0.001^{*} \\ \text { OSI } & 0.001^{*} & 0.999 & 0.001^{*} \\ \text { IMA } & 0.001^{*} & 0.001^{*} & 0.897\end{array}$

*Significant difference, $\mathrm{p}<0.05$

TAS: Total antioxidant status (mmol Trolox Equiv./L); TOS: Total oxidant status ( $\mu$ molH2O2Equiv./L); IMA: Ischemia modified albumin (ABSU); Albumin (g/dL); OSI: Oxidative stress index; and IMAR: IMA-to-serum albumin ratio (arbitrary unit)

and cardiovascular system and intestinal ischemia (13). Moreover, deterioration of the microcirculation due to oxidative stress occurs in the splanchnic region during LC insufflation. Although oxidative stress poses a significant risk due to high 
intraabdominal pressure produced during LC, to the best of our knowledge, this was the first study to investigate the effect of LC performed under different levels of IAP on oxidative stress markers.

The splanchnic circulation results from the gastric, small intestine, colonic, pancreatic, hepatic, and splenic circulation occurring simultaneously. The three major arteries that supply the splanchnic organs, the celiac and superior and inferior mesenteric, give rise to smaller arteries that anastomose extensively. Numerous extrinsic and intrinsic factors influence the splanchnic circulation. Extrinsic factors include the general hemodynamic conditions of the cardiovascular and the autonomic and nervous system and circulating neurohumoral agents. Intrinsic factors include special properties of the vasculature, local metabolites, intrinsic nerves, paracrine substances, and local hormones (14). After an increase in IAP during $L C$, different oxidative stress markers are formed with the contribution of intrinsic and extrinsic factors. $\mathrm{CO}_{2}$ pneumoperitoneum causes significant pathophysiological changes in many systems. The cardiovascular system is the most affected one during laparoscopy. Hypercarbia, acidosis, and an elevated IAP are the main reasons of the observed changes during $\mathrm{CO}_{2}$ pneumoperitoneum (15). Generally, respiratory acidosis is well tolerated, and the blood is normalized by buffering systems. However, in patients with comorbid cardiorespiratory disease and in prolonged surgical procedures with elevated IAP, this recycling system might be challenging or impossible. IMA is formed by the pathophysiological causes of ischemia, such as the free radical formation, hypoxia, acidosis, and a decrease in the binding capacity for transition metals in the $\mathrm{N}$ terminal region of the human albumin (16).

Ischemic events such as pulmonary embolism and myocardial ischemia alter the albumin binding, which may be a marker for ischemia pre-necrosis (10). In the antioxidant defense system developed by the human body, cellular, extracellular, and membrane-like substances are produced to prevent damage by oxidative stress by their reaction with free radicals. The resulting imbalance between the oxidant and antioxidant systems is the main cause of tissue damage.

While low levels of reactive oxygen species such as hydroxyl, peroxyl, and nitric oxide play the role in biological mechanisms such as defense mechanisms against pathogenic microorganisms and intercellular communication, an elevated concentration of such species may cause destruction in DNA, lipids, and proteins, and even cell death (17). Free radicals have the potential to cause damage to all cell components due to reactive structures. It is known that oxidative stress plays a role in the etiopathogenesis of many diseases, such as atherosclerosis, diabetes, cancer, and aging (18). Ortiz-Oshiro et al. (19) suggested that the restriction of pressure by $12 \mathrm{mmHg}$ and a short surgery duration are the key points to prevent potential oxidative injury in laparoscopic surgery.

In another study, an increase in lipid peroxidation products and a decrease in endogenous antioxidants were reported in the early postoperative period in laparoscopic surgery when compared to open cholecystectomy (20). It was reported that the oxidative stress contributed to the pulmonary functions impairment in laparoscopic procedures with $\mathrm{CO}_{2}$ insufflation $(19,20)$.
In a study measuring malondialdehyde in the tissue and blood, TAS, arterial blood gases, blood nitric oxide, and the end-tidal carbon dioxide volume after open and LC, Zulfikaroglu et al. (21) found the level of blood malondialdehyde to be high 30 min into surgery, but no significant changes in TAS. Based on their findings, they concluded that the antioxidant defense system is insufficiently stimulated when faced with a threshold level of oxidative stress. In another study, Koksal et al. (22) found that perioperative and postoperative TOS increased slightly but not significantly in LC patients. In a second study, Koksal et al. (23) found that the IMA values of blood samples taken during LC were higher than those of a control group, a difference that they explained by the improvement in splanchnic ischemia during LC due to PP.

This study tested the hypothesis that changes during LC can be generated by PP in the splanchnic area and the bloodstream of intraabdominal organs at different pressures. To test this hypothesis, albumin, TAS, TOS, OSI, IMA, and IMAR were measured in three patient groups. Oxidative stress markers were compared preoperatively, perioperatively, and postoperatively in the groups. In Group 1, the IMA and IMAR values increased significantly postoperatively, whereas in Group 2, the perioperative TOS and OSI values increased, and a postoperative decrease was observed. All markers showing perioperative oxidative stress, TOS, OSI, and IMA, were found to be significantly elevated in Group 3. At the postoperative 24th hour, the TOS and OSI values decreased significantly, while the IMA elevation continued. This status can be explained by a decrease in the splanchnic blood flow. During LC, high-pressure PP has several side effects, especially on the cardiovascular and pulmonary systems, and high $\mathrm{CO}_{2}$ pressure may lead to acidosis, hypercapnia, deep vein thrombosis, and hypoxia (24).

The major limitation of our study is that the preoperative oxidative stress basal levels of the patients in the groups differ from each other. Differences in the baseline levels of preoperative oxidative stress limit comparing the groups with each other and interpreting the results. Because of this, preoperative, perioperative, and postoperative comparisons were made within the group, and it was observed that a high PP pressure may lead to adverse side effects.

\section{CONCLUSION}

The results of this study indicate that the creation of PP under low pressure during LC produces less oxidative stress than the creation of PP under high pressure. Laparoscopic surgery may alter the oxidative stress and pulmonary function due to IAP. Complications can be avoided with an appropriate anesthetic approach, pneumoperitoneum pressure, and surgical technique.

Ethics Committee Approval: Ethics committee approval was received for this study from the Ethics Committee of Antalya Training and Research Hospital (2013-162).

Informed Consent: Written informed consent was obtained from patient who participated in this study

Peer-review: Externally peer-reviewed.

Author Contributions: Concept - U.D., I.G., M.H., U.K.; Design - U.D., I.G., N.B.; Supervision - U.D., N.B., B.M., M.T.O.; Resource - U.D., H.Y.E., 
B.M.; Materials - U.D., H.Y.E., U.K., T.C.; Data Collection and/or Processing - U.D., T.C., A.A.; Analysis and/or Interpretation - U.D., B.M., M.H.; Literature Search - U.D., U.K., M.T.O.; Writing Manuscript - U.D., M.H., H.Y.E.; Critical Reviews - U.D., B.M., N.B.

Conflict of Interest: The authors have no conflicts of interest to declare.

Financial Disclosure: The authors declared that this study has received no financial support.

\section{REFERENCES}

1. Kama NA, Kologlu M, Doganay M, Reis E, Atli M, Dolapci M. A risk score for conversion from laparoscopic to open cholecystectomy. Am J Surg 2001; 181: 520-525. [CrossRef]

2. McLaughlin JG, Scheeres DE, Dean RJ, Bonnell BW. The adverse hemodynamic effects of laparoscopic cholecystectomy. Surg Endosc 1995; 9: 121-124. [CrossRef]

3. Dexter SP, Vucevic M, Gibson J, McMahon MJ. Hemodynamic consequences of high-and low-pressure capnoperitoneum during laparoscopic cholecystectomy. Surg Endosc 1999; 13: 376381. [CrossRef]

4. Eleftheriadis E, Kotzampassi K, Botsios D, Tzartinoglou E, Farmakis $\mathrm{H}$, Dadoukis J. Splanchnic ischemia during laparoscopic cholecystectomy. Surg Endosc 1996; 10: 324-326. [CrossRef]

5. Glantzounis GK, Tselepis AD, Tambaki AP, Trikalinos TA, Manataki $A D$, Galaris $D A$, et al. Laparoscopic surgery-induced changes in oxidative stress markers in human plasma. Surg Endosc 2001; 15: 1315-1319. [CrossRef]

6. Polat C, YIImaz S, Serteser M, Koken T, Kahraman A, Dilek ON. The effect of different intraabdominal pressures on lipid peroxidation and protein oxidation status during laparoscopic cholecystectomy. Surg Endosc 2003; 17: 1719-1722. [CrossRef]

7. Erel O. A novel automated direct measurement method for total antioxidant capacity using a new generation, more stable ABTS radical cation. Clin Biochem 2004; 37: 277-285. [CrossRef]

8. Erel O. A new automated colorimetric method for measuring total oxidant status. Clin Biochem 2005; 38: 1103-1111. [CrossRef]

9. Harma M, Harma M, Erel O. Increased oxidative stress in patients with hydatidiform mole. Swiss Med Wkly 2003; 133: 563-566.

10. Bar-Or D, Lau E, Winkler JV. A novel assay for cobalt-albumin binding and its potential as a marker for myocardial ischemia-a preliminary report. J Emerg Med 2000; 19: 311-315. [CrossRef]
11. Marschall HU, Einarsson C. Gallstone disease. J Intern Med 2007; 261: 529-542. [CrossRef]

12. Bittner R. The standard of laparoscopic cholecystectomy. Langenbecks Arch Surg 2004; 389: 157-163. [CrossRef]

13. Schilling MK, Redaelli C, Krahenbuhl L, Signer C, Büchler MW. Splanchnic microcirculatory changes during CO2 laparoscopy. J Am Coll Surg 1997; 184: 378-382.

14. Parks DA, Jacobson ED. Physiology of the splanchnic circulation. Arch Intern Med 1985; 145: 1278-1281. [CrossRef]

15. Gutt CN, Oniu T, Mehrabi A, Schemmer P, Kashfi A, Kraus T, et al. Circulatory and respiratory complications of carbon dioxide insufflation. Dig Surg 2004; 21: 95-105. [CrossRef]

16. Sadler PJ, Tucker A, Viles JH. Involvement of a lysine residue in the $\mathrm{N}$-terminal $\mathrm{Ni2}$, and $\mathrm{Cu} 2$ binding site of serum albumins. Comparison with Co2, Cd2, Al3. Eur J Biochem 1994; 220: 193200. [CrossRef]

17. Brown GC, Borutaite V. Nitric oxide, mitochondria, and cell death. IUBMB Life 2001; 52: 189-195. [CrossRef]

18. Neeraj, Pramod J, Singh S, Singh J. Role of free radicals and antioxidants in human health and disease. Int J Curr Res Rev 2013; 5: $14-22$.

19. Ortiz-Oshiro E, Mayol J, Aparicio Medrano JC, Sanjuan Garcia MA, Alvarez Fernandez-Represa J. Lactate metabolism during laparoscopic cholecystectomy: comparison between $\mathrm{CO} 2$ pneumoperitoneum and abdominal wall retraction. World J Surg 2001; 25: 980-984. [CrossRef]

20. Glantzounis GK, Tselepis AD, Tambaki AP, Trikalinos TA, Manataki $A D$, Galaris $D A$, et al. Laparoscopic surgery-induced changes in oxidative stress markers in human plasma. Surg Endosc 2001; 15: 1315-1319. [CrossRef]

21. Zülfikaroğlu B, Koç M, Soran A, Isman FK, Cinel I. Evaluation of oxidative stress in laparoscopic cholecystectomy. Surg Today 2002; 32: 869-874. [CrossRef]

22. Koksal H, Kurban $\mathrm{S}$. Total oxidant status, total antioxidant status, and paraoxonase and arylesterase activities during laparoscopic cholecystectomy. Clinics 2010; 65: 285-290. [CrossRef]

23. Koksal H, Kurban S, Şahin M. The role of ischemia modified albu$\mathrm{min}$ in the evaluation of hemodynamic changes in the splanchnic area during laparoscopic cholecystectomy. Ulus Cerrahi Derg 2010; 26: 91-94. [CrossRef]

24. Goodale RL, Beebe DS, McNevin MP, Boyle M, Letourneau JG, Abrams JH, et al. Hemodynamic, respiratory and metabolic effects of laparoscopic cholecystectomy. Am J Surg 1993; 166: 533537. [CrossRef] 